\title{
Disorder and relaxation mode in the lattice dynamics of the $\mathrm{PbMg}_{1 / 3} \mathrm{Nb}_{2 / 3} \mathrm{O}_{3}$ relaxor ferroelectric
}

\author{
S. N. Gvasaliya* \\ Laboratory for Neutron Scattering, ETHZ \& Paul-Scherrer Institut, CH-5232 Villigen PSI, Switzerland \\ S. G. Lushnikov \\ Ioffe Physical Technical Institute, 26 Politekhnicheskaya, 194021 St. Petersburg, Russia \\ B. Roessli \\ Laboratory for Neutron Scattering, ETHZ \& Paul Scherrer Institut, CH-5232 Villigen PSI, Switzerland
}

(Received 27 August 2003; published 17 March 2004)

\begin{abstract}
The low-energy part of vibration spectrum in $\mathrm{PbMg}_{1 / 3} \mathrm{Nb}_{2 / 3} \mathrm{O}_{3}(\mathrm{PMN})$ relaxor ferroelectric was studied by inelastic neutron scattering. We observed the coexistence of a resolution-limited central peak with strong quasielastic scattering. The line width of the quasielastic component follows a $\Gamma_{0}+D q^{2}$ dependence. We find that $\Gamma_{0}$ is temperature dependent. The relaxation time follows the Arrhenius law well. The presence of a relaxation mode associated with quasielastic scattering in PMN indicates that order-disorder behavior plays an important rôle in the dynamics of diffuse phase transitions.
\end{abstract}

DOI: 10.1103/PhysRevB.69.092105 PACS number(s): 77.80. $-\mathrm{e}, 61.12 .-\mathrm{q}, 63.50 .+\mathrm{x}, 64.60 .-\mathrm{i}$

Enormous attention has been paid to relaxor ferroelectrics for more than 40 years due to their intriguing physical properties. A marking feature observed in relaxors is the frequency-dependent peak in the dielectric permeability $\varepsilon$ which typically extends over a few hundred degrees and does not link directly to the macroscopic changes of symmetry. ${ }^{1}$ Since many physical properties of relaxors exhibit anomalies in this temperature range, it was called "diffuse phase transition. " " Despite intense investigations, however, the nature of the diffuse phase transition in these compounds remains unclear. ${ }^{3-5}$

The well-known crystal $\mathrm{PbMg}_{1 / 3} \mathrm{Nb}_{2 / 3} \mathrm{O}_{3}$ (PMN) is a model relaxor. In PMN, the peak in the real part of the dielectric permeability $\varepsilon^{\prime}$ appears around the mean Curie temperature $T_{c m} \sim 270 \mathrm{~K} .{ }^{2}$ By application of an electric field it is possible to induce a structural phase transition at $T$ $\sim 210 \mathrm{~K} .{ }^{6}$ Furthermore, at $T_{d} \sim 620 \mathrm{~K}$ the temperature dependence of the optical refraction index deviates from the expected linear dependence. ${ }^{7}$ This result was explained by the appearance, far above $T_{m}$, of small polarized regions within the crystal. ${ }^{7}$ This polarization would exist on the scale of a few unit cells ${ }^{7}$ only, and hence called "polar nanoregions" (PNR). The concept of PNR became very popular to account for properties such as the diffuse scattering observed by neutron scattering in relaxors of $\mathrm{PbB}_{1 / 3}^{\prime} \mathrm{B}_{2 / 3}^{\prime \prime} \mathrm{O}_{3}$ type. ${ }^{8-11}$

To obtain a theoretical description of a phase transition, an important point is to know whether this transition is primarily of displacement or of order-disorder type. ${ }^{12}$ Since PMN crystallizes in the perovskite-type structure, it was expected that the diffuse phase transition is of displacement type. Within such a scenario a soft-phonon mode should be observed. Extensive search for the presence of a soft mode in PMN was performed both by light (for a review see Ref. 13) and neutron scattering. From light scattering no soft mode has ever been found. On the other hand, neutron-scattering experiments revealed a very complicated behavior of the phonons in PMN. ${ }^{14-16}$ First, a soft excitation was identified in PMN above $T_{d},{ }^{14}$ which is coupled with transverseacoustic phonon. Later, in Ref. 15 a strong renormalization of the lowest transverse-optic (TO) phonon branch was observed starting from $T \sim 1100 \mathrm{~K}$. Recently, a soft mode coupled to the lowest TO phonon was reported. ${ }^{16}$ Obviously, the identification of a soft mode in PMN has not been definitely settled. In addition to the phonon modes a resolutionlimited central peak $(\mathrm{CP})$ is observed by neutron scattering in PMN without ${ }^{14}$ and with an applied electric field. ${ }^{17}$ The time scale of CP is also at the center of a controversy because it contradicts light-scattering data, ${ }^{18}$ where a broad quasielastic component was observed with a typical width of $2 \mathrm{meV}$. We note that the search for a broad quasielastic component in related relaxor $\mathrm{PbZn}_{1 / 3} \mathrm{Nb}_{2 / 3} \mathrm{O}_{3}+8 \% \mathrm{PbTiO}_{3}$ was not successful $^{19}$ which again is in contradiction with results of light scattering (e.g., Ref. 20). Hence, neutron-scattering data speaks in favor of displacement behavior in relaxor ferroelectrics coexisting with a narrow resolution-limited CP. On the contrary, light-scattering data suggest order-disorder behavior in these compounds.

To solve these discrepancies between light and neutronscattering results and to show that order-disorder behavior plays an important rôle in the lattice dynamics of PMN we have studied the low-energy range of the vibration spectrum in this crystal by inelastic cold-neutron scattering under improved resolution conditions with respect to the previous studies of Refs. 14, 16, and 17. The measurements were carried out with the three-axis spectrometer TASP, located at the neutron spallation source SINQ at the Paul Scherrer Institut, Switzerland. A high-quality single crystal of PMN $\left(\sim 8 \mathrm{~cm}^{3}\right.$, mosaic $\left.\sim 20^{\prime}\right)$ was mounted inside a closed-cycle refrigerator equipped with a small furnace. The crystal was aligned in the $(h h l)$-scattering plane. The measurements were performed in the temperature range $100 \mathrm{~K}-450 \mathrm{~K}$. The (002) reflection of pyrolytic graphite (PG) was used to monochromate and analyze the incident and scattered- 

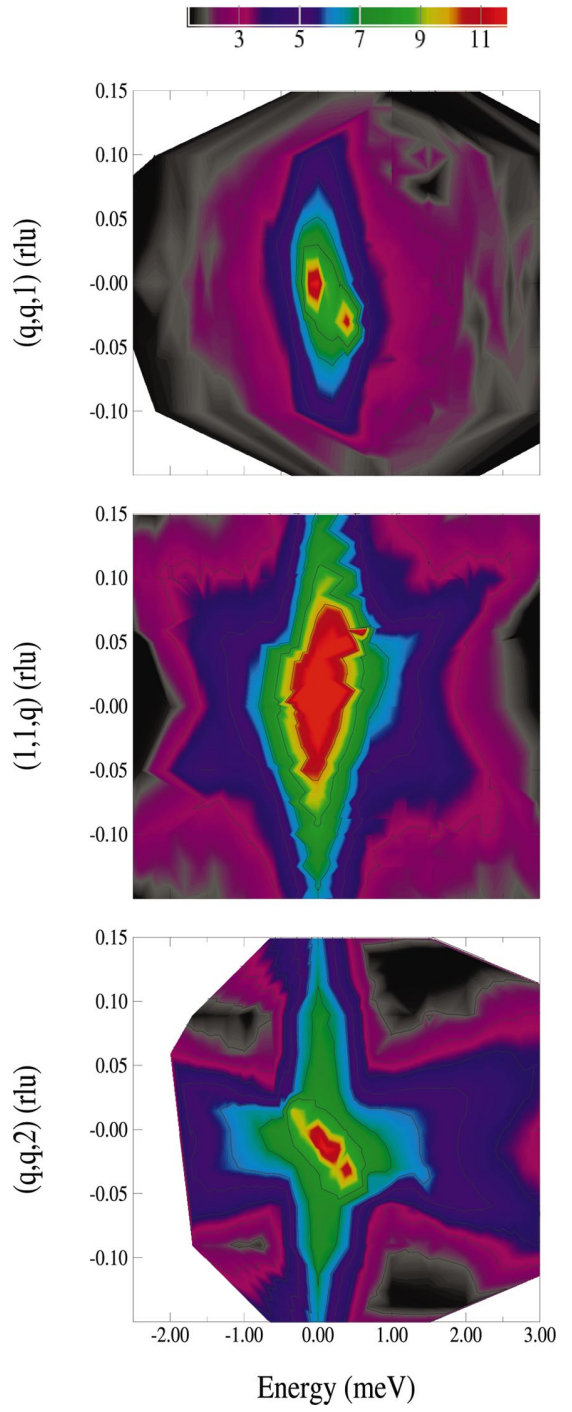

FIG. 1. (Color) Color maps of neutron-scattering intensity around the (001) (top), (110) (middle), and (002) (bottom) Bragg positions at $T=300 \mathrm{~K}$. Asymmetry in the intensity contours of neutron energy gain (negative energies) and neutron energy loss (positive energies) parts of spectra is due to the resolution effects. Note that the intensity is given in a logarithmic scale. $1=$ rlu $=1.555 \AA^{-1}$.

neutron beams, respectively. The spectrometer was operated in the constant final-energy mode with either $k_{f}=1.64 \AA^{-1}$ or $k_{f}=1.97 \AA^{-1}$ using a PG filter to remove higher-order wavelengths. The horizontal collimation was guide $-80^{\prime}$ $-80^{\prime}-80^{\prime}$. With that configuration the energy resolution as measured with a standard vanadium sample is $0.4 \mathrm{meV}$ for $k_{f}=1.97 \AA^{-1}$ and $0.2 \mathrm{meV}$ for $k_{f}=1.64 \AA^{-1}$.

Figure 1 shows $(q, \hbar \omega)$ contour maps of neutronscattering spectra in the vicinity of Bragg positions $\left(\begin{array}{lll}0 & 0 & 1\end{array}\right)$, ( $\left.\begin{array}{lll}1 & 1 & 0\end{array}\right)$, and $\left(\begin{array}{lll}0 & 0 & 2\end{array}\right)$ at $T=300 \mathrm{~K}$, respectively. It is clear that all three figures differ from each other. For example, the intensity distribution in the $\left(\begin{array}{lll}0 & 0 & 1\end{array}\right)$ Brillouin zone (BZ) consists of a Bragg peak, a rather intense signal which is narrow in energy and broad in $q$, and a diffuse signal which is broad both in $q$ and in $\hbar \omega$. In the (llll $\left.\begin{array}{lll}0 & 1\end{array}\right)$ BZ no linear dispersion which could be associated with TA phonons is observed. On the other hand, one can easily see the linear dispersion of TA phonons in the (lo 02 2) BZ, in addition to the Bragg peak and to the intense signal narrow in energy. This signal corresponds to the central peak reported before. ${ }^{14,16}$ Qualitatively, the distribution of the intensity in the (1 100$)$ BZ lies between what is observed in the $\left(\begin{array}{lll}0 & 0 & 1\end{array}\right)$ and $\left(\begin{array}{lll}0 & 0 & 2\end{array}\right)$ zones. The typical peak intensity of a TA phonon measured close to the $\left(\begin{array}{lll}0 & 0 & 2\end{array}\right)$ Brillouin-zone center is $\sim 5000$ counts at room temperature. It is well known that the neutron intensity of an acoustic phonon scales with the structure factor of the corresponding Bragg peak and with the square of the modulus of the scattering vector $Q^{2}{ }^{21}$ The structure factor of the $\left(\begin{array}{lll}0 & 0 & 2\end{array}\right)$ Bragg peak is 100 times larger than that of $\left(\begin{array}{lll}0 & 0 & 1\end{array}\right)$. Also, $Q^{2}$ increases by a factor of $\sim 4$ while measuring in the $\left(\begin{array}{lll}0 & 0 & 2\end{array}\right) \mathrm{BZ}$. Thus, the total decrease of intensity of the TA phonons in $(0$ $\left.\begin{array}{ll}0 & 1\end{array}\right) \mathrm{BZ}$ with respect to $\left(\begin{array}{lll}0 & 0 & 2\end{array}\right)$ amounts to 400 . Hence it is impossible to detect the intensity of such a phonon with respect to the diffuse intensity which amounts to $\sim 200$ counts in the $\left(\begin{array}{lll}0 & 0 & 1\end{array}\right)$ zone.

In this paper we will concentrate on the properties of the quasielastic intensity we found in PMN and not on the behavior of the acoustic phonons nor on the central peak as these have been the subject of numerous studies. ${ }^{8,14-17,22}$ In order to interpret the data quantitatively we decomposed the neutron-scattering spectra $I(\mathbf{Q}, \omega)$ in the following way:

$$
I(\mathbf{Q}, \omega)=\left[S_{C P}+S_{q e l}+S_{D H O}\right] \otimes R(\mathbf{Q}, \omega)+B,
$$

where $S_{C P}$ refers to the resolution-limited central peak; $S_{q e l}$ is the quasielastic scattering shown in Fig. $1 ; S_{D H O}$ describes the phonon scattering. The symbol $\otimes$ stands for the convolution with the spectrometer resolution function $R(\mathbf{Q}, \omega) .{ }^{23} B$ denotes the background level.

We found that the data could be modeled using

$$
\begin{gathered}
S_{C P}=A(q) \delta(\omega), \\
S_{q e l}=I(q) \frac{\omega}{1-\exp (-\omega / T)} \frac{1}{\pi} \frac{\Gamma_{q}}{\omega^{2}+\Gamma_{q}^{2}},
\end{gathered}
$$

and

$$
S_{D H O}=W(q) \frac{\omega}{1-\exp (-\omega / T)} \frac{\gamma_{q}}{\left(\omega^{2}-\Omega_{q}^{2}\right)^{2}+\omega^{2} \gamma_{q}^{2}}
$$

Here $\delta(\omega)$ is the Dirac delta function; $\Gamma_{q}$ and $\gamma_{q}$ are the half width at half maximum (HWHM) of the Lorentzian and damped-harmonic-oscillator function, respectively. $A(q)$, $I(q)$ are the $q$-dependent susceptibilities of the corresponding excitations and $W(q)$ is the TA dynamical structure factor. The renormalized dispersion of the TA phonons is given by $\Omega_{q}=\sqrt{\omega_{q}^{2}+\Gamma_{q}^{2}}$, where $\omega_{q}$ is the frequency of a damped oscillation. In our description, $\Omega_{q}$ is the physically relevant quantity. ${ }^{24}$ Since the measurements are restricted to small values of $q$, we used a linear dispersion for the TA phonon. The validity of such an approximation is clear from the intensity distribution shown in Fig. 1. Figure 2 shows typical constant- $Q$ scans in the $\left(\begin{array}{lll}0 & 0 & 1\end{array}\right)$ and $\left(\begin{array}{lll}1 & 1 & 0\end{array}\right)$ BZ, respectively. 


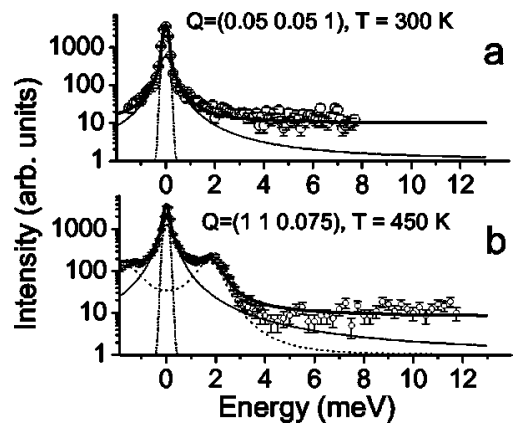

FIG. 2. Typical examples of constant- $Q$ scans in different BZ. The bold line shows the results of data analysis as described in the text. The solid line shows the contribution of the quasielastic component, the dash-dotted line the narrow central peak, and the dotted line the TA phonon.

Comparison between Figs. 2(a) and 2(b) shows that phonons are visible at $\hbar \omega= \pm 1.6 \mathrm{meV}$ for $Q=\left(\begin{array}{lll}1 & 1 & 0.075\end{array}\right)$. On the contrary there is no phonon scattering at $Q=(0.050 .051)$. Hence, to analyze the data in the $\left(\begin{array}{lll}0 & 0 & 1\end{array}\right) \mathrm{BZ}$, we fixed $W(q)$ to zero. The solid lines in Fig. 2 show that the data are well represented by Eq. (1).

Figures 3(a) and 3(b) show the $q$ dependence of the integrated intensity and of the damping of the quasielastic scat-
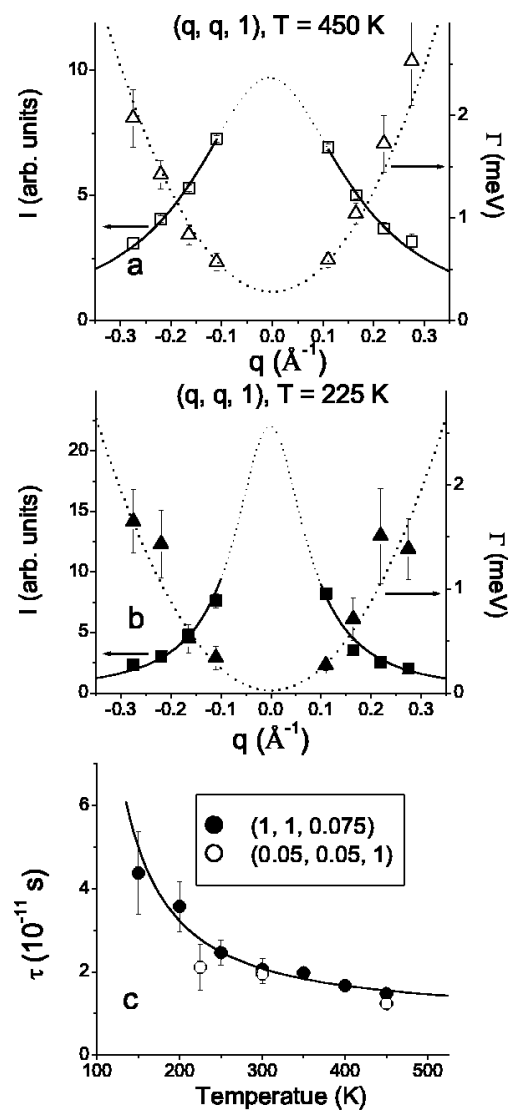

FIG. 3. (a) and (b) $q$ dependence of integrated intensity (squares) and of damping (triangles) of the QE component at $T$ $=450 \mathrm{~K}$ and $225 \mathrm{~K}$, respectively. (c) Temperature dependence of the relaxation time $\tau$ associated with the quasielastic component. The solid line is a fit to the data with the Arrhenius law. tering (QE) observed in the $\left(\begin{array}{lll}0 & 0 & 1\end{array}\right) \mathrm{BZ}$ at $T=450 \mathrm{~K}$ and 225 $\mathrm{K}$. The quasielastic scattering can be approximated by a Lorentzian line shape in $q$ space. Then the correlation length is $\xi=6 \AA$ at $T=450 \mathrm{~K}$ and $\xi=12 \AA$ at $T=225 \mathrm{~K}(\xi$ $=1 / \mathrm{HWHM}$ of the Lorentzian). The damping of the $\mathrm{QE}$ component is found to behave like $\Gamma(q)=\Gamma_{0}+D q^{2}$. At $T$ $=450 \mathrm{~K}$ the parameters are $\Gamma_{0}=0.28 \pm 0.01 \mathrm{meV}$ and $D$ $=24.5 \pm 7.3 \mathrm{meV} \AA^{2}$ and at $T=225 \mathrm{~K}$ we obtain $\Gamma_{0}=0.02$ $\pm 0.01 \mathrm{meV}$ and $D=21.3 \pm 8.9 \mathrm{meV} \AA^{2}$. The absence at all $q$ of an intrinsic energy width of CP implies that the associated fluctuations are very slow. Further, the intensity of the QE scattering does not scale with the narrow CP. In the $\left(\begin{array}{lll}0 & 0 & 2\end{array}\right)$ BZ (Fig. 1 bottom) the CP is very intense, but we were unable to detect $\mathrm{QE}$ component in this BZ. On the contrary, in the $\left(\begin{array}{lll}0 & 0 & 1\end{array}\right) \mathrm{BZ}$ the narrow $\mathrm{CP}$ is significantly weaker and the QE component is clearly seen. The different $q$ dependences of the energy widths and behavior of the relative intensities of the $\mathrm{CP}$ and quasielastic scattering indicate that these processes are of different origins.

We now turn to the temperature behavior of the relaxation time $\tau=1 / \Gamma$ of the QE mode measured in the $\left(\begin{array}{lll}0 & 0 & 1\end{array}\right)$ and $(1,1,0) \mathrm{BZ}$, respectively, and shown in Fig. 3(c). It is obvious that the temperature dependence of $\tau$ is very similar in both zones. We found that $\tau$ is well described by the Arrhenius law $\tau(T)=\tau_{0} \exp \left[E_{a} / k_{B} T\right]$. The solid line in Fig. 3(b) corresponds to the parameters $\tau_{0}=8.6 \pm 0.1 \times 10^{-12} \mathrm{~s}$ and $E_{a}$ $=0.023 \pm 0.0002 \mathrm{eV}$. While these parameters are reasonable, they have to be taken with care as quantities such as the refraction index, ${ }^{7}$ the specific heat, ${ }^{25}$ and the diffuse scattering ${ }^{8,26}$ exhibit anomalous behaviors in this temperature range. This implies that the structure of PMN undergoes significant changes on a local scale, which in turn should affect the value of $E_{a}$. Despite this uncertainty, the fact remains that $\tau(T)$ is connected to a thermally activated behavior, which allows us to speculate about the origin of the relaxation mode.

It is well known that in relaxors $\mathrm{Pb}$ ions are shifted from their ideal positions in the perovskite structure. ${ }^{26}$ Recently it was shown that the probability density function $\rho(R)$ for $\mathrm{Pb}$ in PMN has more than a single maximum below $T_{d} \cdot{ }^{27}$ Therefore, it is reasonable to assume that at high temperature the $\mathrm{Pb}$ ions move between maxima of $\rho(R)$. In that case when the temperature is low enough with respect with the potential barrier, the hopping frequency $1 / \tau$ becomes small. This gives rise to a narrowing in the energy width of the $\mathrm{QE}$ component.

There are a number of models which describe the presence of a central peak in the vicinity of a structural phase transition (for a review of earlier papers see Ref. 28). However, PMN does not undergo any structural phase transition without application of an electric field. To link the coexistence of a central peak with quasielastic scattering in a broad temperature range might be of importance to understand the diffuse phase transition in relaxor ferroelectrics.

To summarize, we have carried out high-resolution neutron-scattering measurements in the relaxor ferroelectric PMN. The low-energy spectra exhibit two components associated with two different time scales. One is the resolution- 
limited central peak. The other component is quasielastic and appears to be related to ionic motion of thermally activated character. The damping of this relaxation mode follows a $q^{2}$ dependence. The presence of a relaxation mode associated with quasielastic scattering in PMN indicates that orderdisorder behavior plays an important rôle in the dynamics of diffuse phase transitions. It will be interesting to see if this relaxation mode, which was also observed in
$\mathrm{PbMb}_{113} \mathrm{Ta}_{213} \mathrm{O}_{3},{ }^{11}$ is a common feature of all relaxor ferroelectrics.

The authors would like to thank Professor R.A. Cowley for invaluable discussions. This work was performed at the spallation neutron source SINQ, Paul Scherrer Institut, Villigen (Switzerland) and was partially supported by RFBR Grant No. 02-02-17678, and by Presidental Grant No. RF ss-1415.2003.2.
*On leave from Ioffe Physical Technical Institute, 26 Politekhnicheskaya, 194021 St. Petersburg, Russia.

${ }^{1}$ L.E. Cross, Ferroelectrics 76, 241 (1987).

${ }^{2}$ G.A. Smolenskii et al., Ferroelectrics and Related Materials (Gordon and Breach, New York, 1984).

${ }^{3}$ V. Westphal, W. Kleemann, and M.D. Glinchuk, Phys. Rev. Lett. 68, 847 (1992).

${ }^{4}$ D. Viehland, S.J. Jang, L.E. Cross, and M. Wuttig, Phys. Rev. B 46, 8003 (1992).

${ }^{5}$ R. Blinc, J. Dolinsek, A. Gregorovic, B. Zalar, C. Filipic, Z. Kutnjak, A. Levstik, and R. Pirc, Phys. Rev. Lett. 83, 424 (1999).

${ }^{6}$ G. Schmidt, H. Arndt, J. Voncieminski, T. Petzsche, H. Voigt, and N. Krainik, Krist. Tech. 15, 1415 (1980).

${ }^{7}$ G. Burns and B.A. Scott, Solid State Commun. 13, 423 (1973).

${ }^{8}$ K. Hirota, Z.-G. Ye, S. Wakimoto, P.M. Gehring, and G. Shirane, Phys. Rev. B 65, 104105 (2002).

${ }^{9}$ S. Wakimoto, C. Stock, Z.-G. Ye, W. Chen, P.M. Gehring, and G. Shirane, Phys. Rev. B 66, 224102 (2002).

${ }^{10}$ D. La-Orauttapong, J. Toulose, J. Robertson, and Z.-G. Ye, Phys. Rev. B 64, 212201 (2001).

${ }^{11}$ S.N. Gvasaliya, B. Roessli, and S. G Lushnikov, Europhys. Lett. 63, 303 (2003).

${ }^{12}$ R.A. Cowley, Adv. Phys. 29, 1 (1980).

${ }^{13}$ I.G. Siny, S.G. Lushnikov, R.S. Kariyar, and V.H. Schmidt, Ferroelectrics 226, 191 (1997).

${ }^{14}$ A.A. Naberezhnov, S.B. Vakhrushev, B. Dorner, D. Stauch, and H. Moudden, Eur. Phys. J. B 11, 13 (1999).
${ }^{15}$ P.M. Gehring, S. Wakimoto, Z.-G. Ye, and G. Shirane, Phys. Rev. Lett. 87, 277601 (2001).

${ }^{16}$ S.B. Vakhrushev and S.M. Shapiro, Phys. Rev. B 66, 214101 (2002).

${ }^{17}$ Y.O. Chetverikov, A.A. Naberezhnov, S.B. Vakhrushev, B. Dorner, and A.S. Ivanov, Appl. Phys. A: Mater. Sci. Process. A74, S989 (2002).

${ }^{18}$ I.G. Siny, S.G. Lushnikov, R. Katiyar, and E. Rogacheva, Phys. Rev. B 56, 7962 (1997).

${ }^{19}$ J. Hlinka, S. Kamba, J. Petzelt, J. Kulda, C.A. Randall, and S.J. Zhang, J. Phys.: Condens. Matter 15, 4249 (2003).

${ }^{20}$ F. Jiang and S. Kojima, Ferroelectrics 266, 19 (2002).

${ }^{21}$ See, e.g., S.W. Lovesey, Thermal Neutron Scattering (Oxford University Press, Oxford, UK, 1984).

${ }^{22}$ S. Wakimoto, C. Stock, R.J. Birgeneau, Z.-G. Ye, W. Chen, W.J.L. Buyers, P.M. Gehring, and G. Shirane, Phys. Rev. B 65, 172105 (2002).

${ }^{23}$ M. Popovichi, Acta Crystallogr., Sect. A: Cryst. Phys., Diffr., Theor. Gen. Crystallogr. A31, 507 (1975).

${ }^{24}$ B. Fåk and B. Dorner, Physica B 234, 1107 (1997).

${ }^{25}$ Y. Moriya, H. Kawaji, T. Tojo, and T. Atake, Phys. Rev. Lett. 90, 205901 (2003).

${ }^{26}$ B. Dkhil, J.M. Kiat, G. Calvarin, G. Baldinozzi, S.B. Vakhrushev, and E. Suard, Phys. Rev. B 65, 024104 (2002).

${ }^{27}$ S.B. Vakhrushev and N.M. Okuneva, in Fundamental Physics of Ferroelectrics 2002, edited by Ronald E. Cohen, AIP Conf. Proc. 626 (AIP, Melville, NY, 2002), p. 117.

${ }^{28}$ A.D. Bruce and R.A. Cowley, Adv. Phys. 29, 219 (1980). 